\title{
Early Spontaneous Resolution of an latrogenic Acute Type A Aortic Dissection
}

\author{
Mohammad A. Zafar, MBBS ${ }^{1}$, Philip Y. K. Pang, MD ${ }^{1,2}$, Glen A. Henry, MD², \\ Bulat A. Ziganshin, MD ${ }^{1,4}$, Maryann Tranquilli, RN'1, John A. Elefteriades, MD ${ }^{*}$ \\ 1 Aortic Institute at Yale-New Haven Hospital, Yale University School of Medicine, New Haven, Connecticut, USA \\ 2 Department of Cardiothoracic Surgery, National Heart Centre, Singapore \\ 3 Department of Internal Medicine, Section of Cardiovascular Medicine, Yale University School of Medicine, New Haven, Connecticut, USA \\ 4 Department of Surgical Diseases \#2, Kazan State Medical University, Kazan, Russia
}

\begin{abstract}
Acute aortic dissection is a rare but devastating complication during cardiac catheterization. We present the case of an elderly female who incurred a Stanford Type A/DeBakey Type I acute aortic dissection extending into the arch vessels and descending aorta likely occurring during right coronary artery engagement for angioplasty. The patient was treated successfully by immediately sealing the entrance of the dissection via the placement of a stent and anti-impulse therapy. Follow-up computed tomography scan showed complete resolution of the dissection within one month.
\end{abstract}

\section{Key Words}

latrogenic aortic dissection - Angioplasty • Percutaneous coronary intervention - Complication

Copyright (c) 2016 Science International Corp.

\section{Introduction}

Acute Stanford Type A aortic dissection (AAD) is a catastrophic medical emergency associated with a high (57\%) early mortality without surgical intervention [1]. latrogenic AAD (IAAD) may rarely present as a complication during coronary angioplasty [2-3]. We



Fax +1 2037853552

E-Mail: aorta@scienceinternational.org

http://aorta.scienceinternational.org

\author{
(c) 2016 AORTA \\ Published by Science International Corp. \\ ISSN 2325-4637 \\ Accessible online at: \\ http://aorta.scienceinternational.org
}

present the case of an IAAD complicating right coronary artery (RCA) angioplasty, for which the entry site of the dissection was stented successfully. Aggressive anti-impulse therapy was instituted, resulting in near complete resolution of the IAAD within 5 days and complete healing within one month.

\section{Case Presentation}

A 71-year-old female with hypertension and hyperlipidemia was investigated for symptoms of worsening exertional dyspnea and chest discomfort. A nuclear cardiac stress test revealed moderate focal inferobasal ischemia. Subsequent diagnostic coronary angiography via the right radial artery revealed a chronic total occlusion of the distal right coronary artery (RCA), with left to right collaterals to the posterior descending artery and posterolateral branches. There was no significant coronary artery disease affecting the left coronary system. Percutaneous coronary intervention ( $\mathrm{PCl}$ ) was then attempted on the distal RCA using an Amplatz (AL1) guide catheter but it was too large and the RCA was not engaged. A Judkins (JR4) guide catheter was then employed to engage the RCA and a FineCross and Fielder FC guidewire was passed to the distal RCA. At this point a large aortocoronary dissection was noted on angi-

* Corresponding Author:

John A. Elefteriades, MD

Aortic Institute at Yale-New Haven

Yale University School of Medicine

789 Howard Avenue, Clinic Building CB 317, New Haven, CT 06510, USA

Tel. +1 203785 2551, Fax: +1 203785 3552, E-Mail: john.elefteriades@yale.edu 

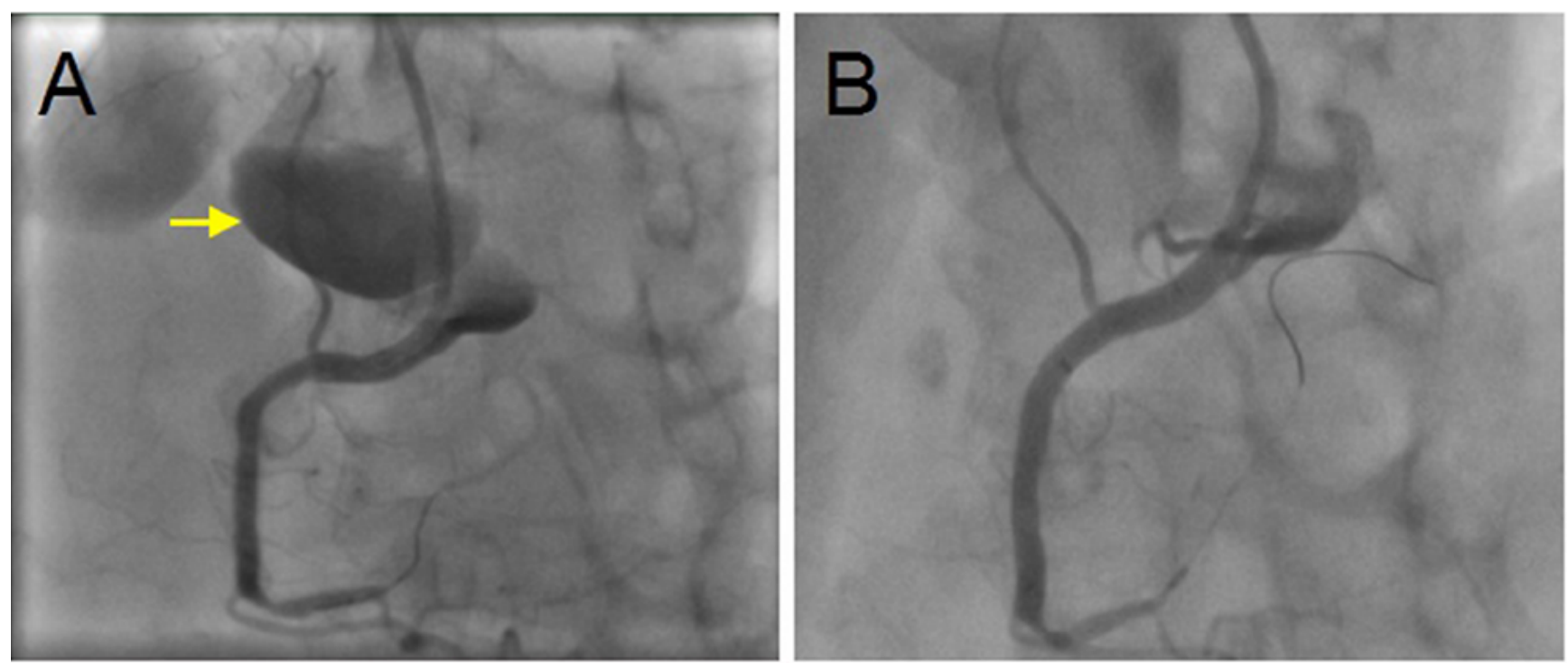

Figure 1. Coronary angiography. Panel A. Dissection of the right coronary artery (RCA) ostium causing retrograde dissection and retention of contrast media within the false lumen of the ascending aorta (arrow). Panel $B$. The entry site of the dissection was sealed successfully by stenting across the proximal RCA into the ostium.

ography (Figure 1). The dissection involved the RCA ostium and proximal RCA, extending to the thoracic aorta. Upon recognition of this severe complication, a coronary stent was promptly placed across the entry site of the dissection, traversing the RCA ostium and proximal RCA with a small protrusion into the aorta. Subsequent contrast injections were minimized and a Guidezilla ${ }^{\mathrm{TM}}$ was inserted to be sure that all contrast injections were well past any possible entry point of the dissection. Intravascular ultrasound showed good stent apposition, complete coverage through the RCA ostium and no evidence of flow outside of the stent. The patient's hemodynamics remained stable throughout the procedure.

An urgent transthoracic echocardiogram showed no pericardial effusion, normal left ventricular ejection fraction, mild central aortic insufficiency and evidence of ascending aortic hematoma. Computed tomographic angiography (CTA) of the chest confirmed the diagnosis of a Stanford Type A aortic dissection starting at the origin of the RCA, extending to the proximal descending thoracic aorta, at the level of the right pulmonary artery. The false lumen extended to the non-coronary cusp but spared the
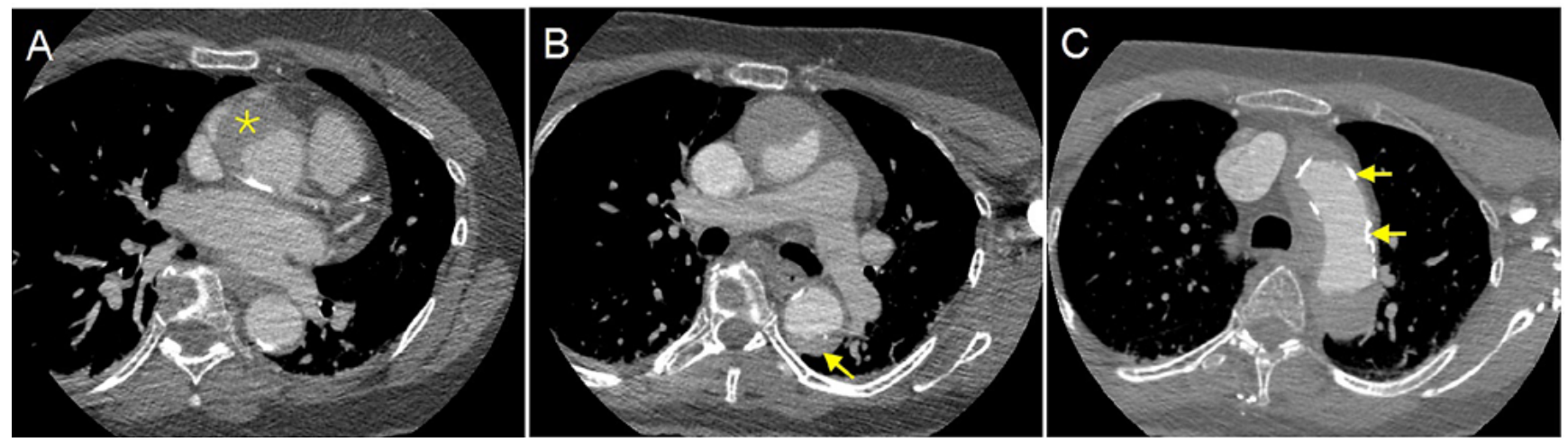

Figure 2. Computed tomographic scan of the chest on Day 1. Panel A. Transverse view at the level of the left coronary ostium showing the false lumen (asterisk) involving the right and non-coronary sinuses but sparing the left coronary sinus. Panel B. Transverse view at the level of the main pulmonary arteries showing the dissection extending to the descending thoracic aorta (arrow). Panel $C$. Transverse view of the aortic arch showing extensive aortic dissection and calcifications within the aortic wall (arrows). 

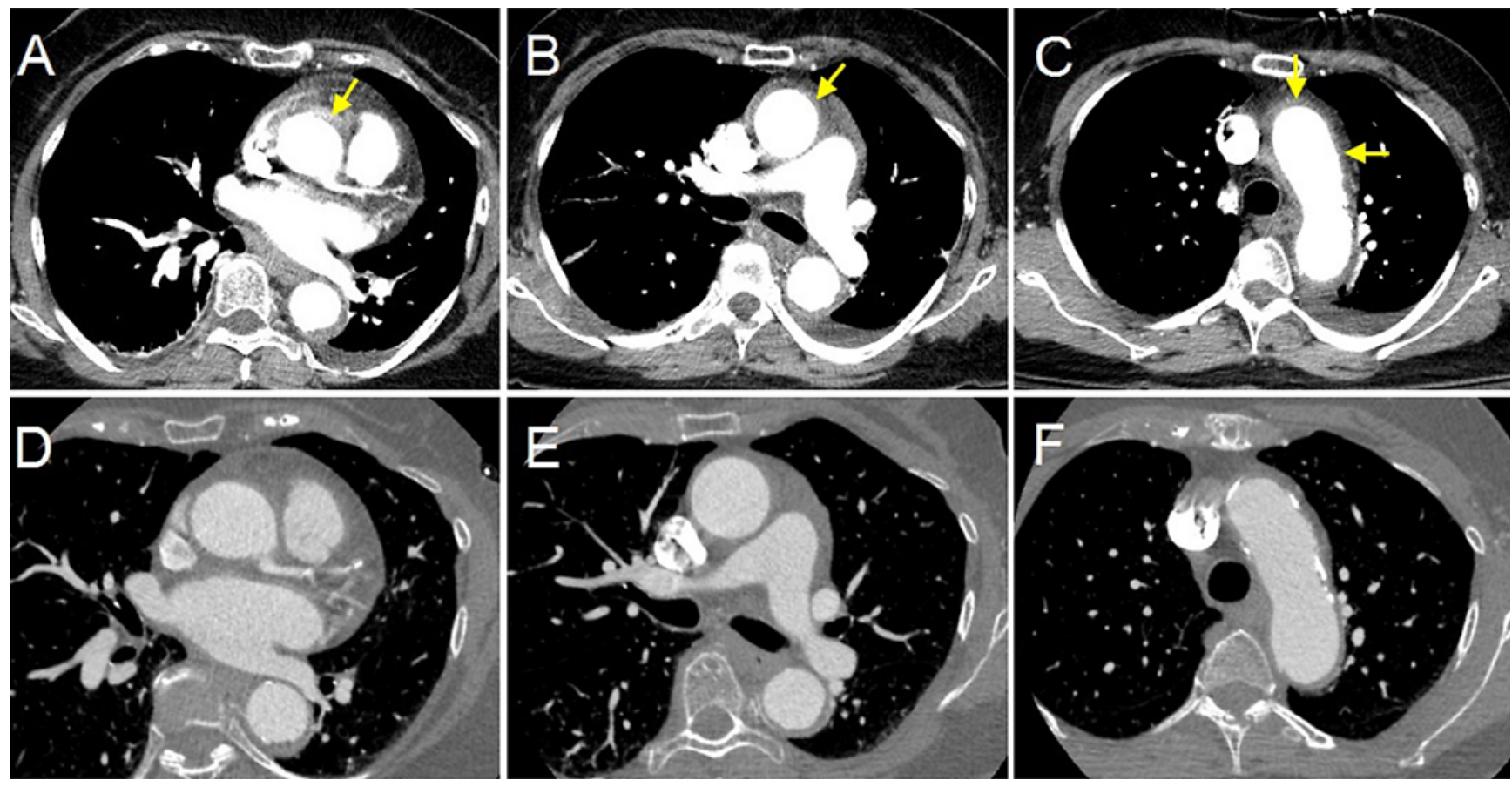

Figure 3. Computed tomographic scan of the chest on Day 5 (Panels A-C) and 1-month post-dissection (Panels D-F). Transverse views at the level of the left coronary ostium (Panel A), main pulmonary arteries (Panel B) and aortic arch (Panel C), showing near-complete resolution of aortic dissection with minimal residual intramural hematoma (arrows). Axial views at the level of the left coronary ostia (Panel D), main pulmonary arteries (Panel E) and aortic arch (Panel F) showing complete resolution of the dissection.

left coronary cusp (Figure 2). The dissection also extended into the right brachiocephalic and right common carotid arteries, with non-critical stenosis of the proximal right common carotid artery. The ascending aorta was aneurysmal, measuring $4.4 \mathrm{~cm}$ maximally in the mid-ascending portion. The descending thoracic aorta measured $3.3 \mathrm{~cm}$ at its widest in the proximal descending portion distal to the left subclavian artery. A very heavily calcified ring in the aortic wall, just above the RCA ostium, likely increased the risk of aortic dissection during angioplasty substantially.

On examination, the patient was in no acute pain or distress. She was hemodynamically stable with no neurological deficit. She was admitted to our intensive care unit for aggressive anti-impulse therapy and close monitoring [4]. Conservative management was favored over surgical intervention on the aortic root because of several technical considerations. Firstly, the proximal aortic anastomosis in the vicinity of the large calcified ring would be very difficult and precarious. Secondly, it would be dangerous handling the RCA with the stent extending through the ostium.
Thirdly, the significant amount of calcium in the aortic arch would pose a very high embolic risk. We were hoping that intensive anti-impulse therapy would limit the progression of this dissection and promote thrombosis of the false lumen.

Serial transthoracic echocardiograms performed during her hospital stay showed interval stability without evidence of pericardial effusion. CTA performed 4 days after her initial scan demonstrated interval resolution of the aortic dissection (Figure $3 \mathrm{~A}-\mathrm{C}$ ), with minimal intramural hematoma extending from the aortic root to the mid descending thoracic aorta. The maximal ascending aortic diameter had decreased to $4.2 \mathrm{~cm}$ and the maximal descending thoracic aortic diameter had decreased to $3.1 \mathrm{~cm}$. She remained pain-free throughout her hospital stay and was discharged in stable condition after 6 days. A follow-up CTA one month later showed complete resolution of the dissection (Figure 3D-F). She was asymptomatic with normal effort tolerance and her physical examination was unremarkable. 


\section{Discussion}

The incidence of IAAD arising as a complication of coronary catheterization ranges from $0.02 \%$ to $0.06 \%$ $[2,3]$. In challenging cases involving $\mathrm{PCl}$ to chronically occluded vessels, the risk of IAAD has been reported to be $1.9 \%$ [5]. Despite the potentially devastating nature of this complication, no clear guidelines exist regarding its optimal management. Dunning et al. [2] previously described a classification system for these dissections based on the degree of aortic involvement: Class 1 included dissections limited to the coronary cusp; Class 2 included dissections extending less than $40 \mathrm{~mm}$ up the ascending aorta whereas Class 3 included those extending distally for more than 40 $\mathrm{mm}$. The authors recommended treating Class 1 and 2 lesions with stenting and/or medical therapy, and Class 3 surgically. Surgical management is the treatment of choice for spontaneous AAD [1]. However, emergent surgical correction of IAAD following $\mathrm{PCl}$ is particularly challenging due to concomitant anticoagulation used during $\mathrm{PCl}$ and varying degrees of myocardial ischemia resulting from co-existing coronary artery disease.

Various reports have described the merits of treating even extensive PCI-related IAADs non-surgically, with coronary stenting and/or conservative management $[3,6,7]$. In a series of 74 patients with cardiac catheterization induced aortocoronary dissections (20\% with Dunning Class 3 dissections), Núñez-Gil et al. [3] demonstrated good results of stenting and/ or conservative therapy, with 69 patients (93\%) remaining complication-free at a median follow-up of 5 years. Only 3 patients (4\%) required cardiac surgery and $2(3 \%)$ succumbed to sequelae of aortic dissection during early follow-up. Follow-up imaging revealed a completely healed aorta, similar to our case, in 5 patients $(7 \%)$.

The importance of abrogating the propagation of the dissection by sealing its entry point in the coronary artery via stenting have been highlighted in previous reviews of catheter-induced aortocoronary dissections [6, 7]. Shah et al. [6] described a series of 86 patients, of whom 65 patients $(76 \%)$ underwent stenting and/or conservative therapy with 3 deaths (3\%). The remaining 21 patients (24\%) underwent aortic repair with or without coronary artery bypass grafting. Amongst those who received surgical treatment, 3 patients (14\%) died. Carstensen et al. [7] reported a series of 67 patients, of whom 55 (82\%) underwent stenting and/or conservative therapy. Two deaths (4\%) occurred in the group treated non-surgically and 3 deaths ( $25 \%$ ) occurred within the surgically treated group.

The majority of patients with spontaneous AAD have inherently diseased aortic walls. In contrast, apart from age-related changes, patients with IAAD may have relatively normal aortic walls, which could possibly permit better healing once the entry site of the aortic dissection is sealed. Also, the presence of advanced atherosclerosis could limit the dissection plane. This may explain the tendency for patients with catheter-induced IAAD to respond well to stenting and/or conservative therapy.

This case highlights the role of non-surgical management of IAAD, especially in the setting of high-risk surgical candidates with unfavorable anatomy or severe comorbidities. Despite the impressive nature of our patient's dissection, there was no compromised perfusion, significant aortic insufficiency, or evolving pericardial effusion allowing meticulous observation and aggressive medical management thereby avoiding a very high risk surgery. There was a rapid recovery with spontaneous improvement beginning within days and complete resolution by CT at one month.

\section{Conflict of Interest}

The authors have no conflict of interest relevant to this publication.

Comment on this Article or Ask a Question 


\section{References}

1. Pape LA, Awais M, Woznicki EM, Suzuki T, Trimarchi S, Evangelista A, et al. Presentation, Diagnosis, and Outcomes of Acute Aortic Dissection: 17-Year Trends From the International Registry of Acute Aortic Dissection. Journal of the American College of Cardiology. 2015;66:350-358. DOI: 10.1016/j.jacc.2015.05.029

2. Dunning DW, Kahn JK, Hawkins ET, O'Neill WW. latrogenic coronary artery dissections extending into and involving the aortic root. Catheterization and Cardiovascular Interventions. 2000;51:387-393. DOI: 10.1002/1522$726 \times(200012) 51: 4<387:: A$ ID CCD3>3.0.CO;2-B

3. Núñez-Gil IJ, Bautista $D$, Cerrato E, Salinas $P$, Varbella $F$, Omedè $P$, et al. Incidence, Management, and Immediate- and Long-Term Outcomes After latrogenic Aortic Dissec- tion During Diagnostic or Interventional Coronary Procedures CLINICAL PERSPECTIVE. Circulation. 2015;131:2114-2119. DOI: 10.1161/CIRCULATIONAHA.115.015334

4. Feldman M, Shah M, Elefteriades JA. Medical Management of Acute Type A Aortic Dissection. Annals of Thoracic and Cardiovascular Surgery. 2009;15:286-293. PMID: 19901881

5. El Sabbagh A, Patel VG, Jeroudi OM, Michael TT, Alomar ME, Mogabgab O, et al. Angiographic success and procedural complications in patients undergoing retrograde percutaneous coronary chronic total occlusion interventions: A weighted meta-analysis of 3482 patients from 26 studies. International Journal of Cardiology. 2014;174:243-248. DOI: 10.1016/j.ijcard.2014.04.004

6. Shah P, Bajaj S, Shamoon F. Aortic Dissec- tion Caused by Percutaneous Coronary Intervention: 2 New Case Reports and Detailed Analysis of 86 Previous Cases. Texas Heart Institute Journal. 2016;43:52-60. DOI: 10.14503/THIJ-14-4585

7. Carstensen S, Ward MR. latrogenic aortocoronary dissection: The Case for Immediate Aortoostial Stenting. Heart, Lung and Circulation. 2008;17:325-329. DOI: 10.1016/j.hlc.2007.11.140.

Cite this article as: Zafar MA, Pang PYK, Henry GA, Ziganshin BA, Tranquilli $M$, Elefteriades JA. Early Spontaneous Resolution of an latrogenic Acute Type A Aortic Dissection. AORTA (Stamford). 2016;4(6):235-239. DOI: http://dx.doi. org/10.12945/j.aorta.2016.16.067 